\title{
Why is $d$-wave pairing in HTS robust in the presence of impurities?
}

\author{
Miodrag L. Kulić \\ Max-Planck-Institut für Festkörperforschung, 70569 Stuttgart, Germany \\ Viktor Oudovenko \\ Max-Planck-Institut für Festkörperforschung, 70569 Stuttgart, Germany \\ Joint Institute for Nuclear Research, 141980 Dubna, Russia
}

(14 January 1997)

\begin{abstract}
In the recent theory of strong correlations by [1] - [3] it was shown that by lowering doping concentration a forward peak is developed in the charge scattering channel. Accordingly, near the optimal doping the nonmagnetic scattering is pronounced in the $d$-channel and $d$-wave pairing is robust against defects and impurities. It is reflected in a decrease of the slope of the critical temperature $T_{c}\left(\Gamma_{s}\right)$ at $\Gamma_{s} \rightarrow 0$ by factor $(1-\beta)$, where $\beta$ is the scattering anisotropy parameter and $\Gamma_{s}$ is an average scattering rate. For large doping, $\beta$ is small [1], [2] and $d-$ wave pairing loses its robustness. The theory is generally formulated for the bi-layer model by including: (1) intra- and inter-plane pairing; (2) intra- and inter-plane impurities.
\end{abstract}

Recently there were a lot of evidences for $d$-wave pairing in high- $T_{c^{-}}$-superconductors $(H T S)$. However, the mechanism of pairing is still unknown, although many proposals were done. For instance, there were claims that superconductivity is due to strong correlations [ [1, or that $A F$ fluctuations are important - see [5]. Even the electron-phonon interaction renormalized by strong correlations can give rise to $d$ - wave pairing, as it was shown by Kulić and Zeyher [1], [2], [3]. Moreover, in [1], [2], [3] it was shown that the charge-charge scattering is renormalized via a charge-vertex $\gamma(\vec{q})$ (screening due to strong correlations) with a peak in the forward scattering $\left(|\vec{q}| \ll k_{F}\right)$, while the backward scattering (large $|\vec{q}|$ ) is suppressed. This means that a bare nonmagnetic scattering potential $U_{0}(\vec{q})$ is renormalized by strong correlations $U_{0}(\vec{q}) \rightarrow U_{\text {eff }}(\vec{q})$. Note, in the Born approximation (after averaging over impurity positions) one has $U_{\text {eff }}(\vec{q})=\gamma^{2}(\vec{q}) u_{0}^{2}(\vec{q}) \equiv u^{2}(\vec{q})$, where $u_{0}(\vec{q})$ is the single impurity potential. It follows [1], [2], [3] that when the bare potential is local, i.e. $u_{0}^{2}(\vec{q})=$ const, $U_{\text {eff }}$ becomes "long-ranged" in the presence of strong correlations. Moreover, within the $t-J$ model [1], [2], [3] and at some optimal doping $\delta \approx 0.2$ the $d$-channel component of $U_{\text {eff }}(\vec{q})$ is of the same order of magnitude as the one in the fully symmetric $s$-channel. When $U_{0}(\vec{q})$ is due to the electron-phonon interaction then superconductivity can be also of $d$-type in the presence of strong Hubbard repulsion.

If we accept that superconductivity in HTS is of $d$ - wave type, then one can put a question: why is $d$-wave pairing robust in the presence of various kinds impurities and defects in HTS materials, as it was observed experimentally [6], [7]? In this paper we present a simple theory, which is based on the effective nonmagnetic impurity potential $U_{\text {eff }}(\vec{q})$ with the large $d$-component and investigate its effect on $d$-wave pairing [8]. Experiments on the irradiation of samples as well as on substitution of atoms in HTS materials are analyzed by using the proposed theory. In the following we consider the weak coupling theory [9] and its extension to the bi-layer systems [10], [11]. In the bi-layer model (adequate for $\mathrm{YBa}_{2} \mathrm{Cu}_{3} \mathrm{O}_{7-x}$ ) with the hopping $t_{\perp}$ between two layers (separated by $Y$ ions) and with the intra- and inter-layer pairings $\Delta_{\|}(\vec{p})$ and $\Delta_{\perp}(\vec{p})$ respectively the averaged (over impurity positions) Green's function $\hat{G}(p)\left(\equiv \hat{G}\left(\vec{p}, i \omega_{n}\right)\right)$ is $4 \times 4$ matrix. It is given by $\hat{G}^{-1}(p)=\hat{G}_{0}^{-1}(p)-\hat{\Sigma}_{i m p}(p) \hat{G}(p)$, where $\hat{G}_{0}(p)$ is the bare Green's function and the impurity self-energy matrix $\hat{\Sigma}_{i m p}(p)$ in the bi-layer model is given by [9]

$$
\hat{\Sigma}_{i m p}\left(\vec{p}, i \omega_{n}\right)=c \int \frac{d^{2} p^{\prime}}{(2 \pi)^{2}} \hat{U}_{e f f}^{i m p}\left(\vec{p}, \vec{p}^{\prime}\right) \hat{G}\left(\vec{p}^{\prime}, i \omega_{n}\right) .
$$

Here, $\omega_{n}=\pi T(2 n+1)$ and $c$ are Matsubara frequencies and the concentration of impurities respectively. In order to simplify notations we set $\hat{U}_{\text {eff }}^{i m p}\left(\vec{p}, \vec{p}^{\prime}\right) \equiv \hat{U}\left(\vec{p}, \vec{p}^{\prime}\right)$. The structure of the $4 \times 4$ matrix $\hat{U}\left(\vec{p}, \vec{p}^{\prime}\right)$ depends on the type of impurities: Case $I$ - there are inter-layer impurities (for instance substitution of $Y$ by Pr); Case $I I$ there are intra-layer impurities (for instance substitution of $C u$ by $Z n$, or the presence of oxygen vacancies due to ion damaging etc.). They are explicitly given in [10] - see also below. Since, in the weak coupling limit the relevant processes are taking place near the Fermi surface it is assumed that the matrix $\hat{U}\left(\vec{p}, \vec{p}^{\prime}\right) \equiv \hat{U}\left(\theta, \theta^{\prime}\right)$ depends on the angles $\theta, \theta^{\prime}$ of momenta on the Fermi surface. In the following, the definition $\int_{0}^{2 \pi} \frac{d \theta^{\prime}}{2 \pi}(\ldots) \equiv\langle(\ldots)\rangle_{\theta^{\prime}}$ is used. $\hat{G}^{-1}\left(\vec{p}, i \omega_{n}\right)$ in the bi-layer model is searched in the form analogous to that given in [10], [11], with renormalized Matsubara frequencies $\tilde{\omega}_{n}$ and renormalized superconducting order parameters $\tilde{\Delta}_{n, i}(\vec{p})(i=1,2$ is the band index -see below)

$$
\tilde{\omega}_{n}(\theta)=\omega_{n}+\frac{1}{2}\left\langle\tau_{\bar{i} \bar{i}}^{-1}\left(\theta, \theta^{\prime}\right) \frac{\tilde{\omega}_{n}\left(\theta^{\prime}\right)}{\sqrt{\tilde{\omega}_{n}^{2}\left(\theta^{\prime}\right)+\tilde{\Delta}_{n, \bar{i}}^{2}\left(\theta^{\prime}\right)}}\right\rangle_{\theta^{\prime}}
$$




$$
\tilde{\Delta}_{n, i}(\theta)=\Delta_{i}(\theta)+\frac{1}{2}\left\langle\tau_{i \bar{j}}^{-1}\left(\theta, \theta^{\prime}\right) \frac{\tilde{\Delta}_{n, \bar{j}}\left(\theta^{\prime}\right)}{\sqrt{\tilde{\omega}_{n}^{2}\left(\theta^{\prime}\right)+\tilde{\Delta}_{n, \bar{j}}^{2}\left(\theta^{\prime}\right)}}\right\rangle_{\theta^{\prime}} .
$$

To this set of equations one should add also the selfconsistent gap equations

$$
\Delta_{i}(\theta)=T_{c} \sum_{n}\left\langle\lambda_{i \bar{j}}\left(\theta, \theta^{\prime}\right) \frac{\tilde{\Delta}_{n, \bar{j}}\left(\theta^{\prime}\right)}{\sqrt{\tilde{\omega}_{n}^{2}\left(\theta^{\prime}\right)+\tilde{\Delta}_{n, \bar{j}}^{2}\left(\theta^{\prime}\right)}}\right\rangle_{\theta^{\prime}} .
$$

The summation over the bar indices is assumed everywhere. Here, $\Delta_{i}(\theta)(i=1,2)$ is the gap in the $i-t h$ band, i.e. $\Delta_{1,2}(\theta)=\Delta_{\|}(\vec{p}) \mp \Delta_{\perp}(\vec{p})$ where $\lambda_{i j}\left(\theta, \theta^{\prime}\right)$ $(i, j=1,2)$ are the intra- and inter-band pairing coupling constants 10]. In the following, we assume $d$-wave pairing (instead of the $s$-wave one used in [10]) $\Delta_{i}(\theta)=$ $\Delta_{i} Y_{d}(\theta)=-\Delta_{i}(\theta+\pi / 2),\left\langle Y_{d}(\theta)\right\rangle_{\theta}=0,\left\langle Y_{d}^{2}(\theta)\right\rangle_{\theta}=1$, and $\lambda_{i j}\left(\theta, \theta^{\prime}\right)=\lambda_{i j} Y_{d}(\theta) Y_{d}\left(\theta^{\prime}\right)$. Near the critical temperature $T_{c}$ one can linearize eqs.(2)-(4) which gives

$$
\begin{gathered}
\tilde{\omega}_{n}(\theta)=\omega_{n}\left[1+\frac{1}{2} \frac{\left\langle\tau_{\bar{i}}^{-1}\left(\theta, \theta^{\prime}\right)\right\rangle_{\theta^{\prime}}}{\left|\omega_{n}\right|}\right] \equiv \omega_{n} \eta_{n}(\theta) \\
\tilde{\Delta}_{n, i}(\theta)=\eta_{n, i \bar{j}}(\theta) \Delta_{\bar{j}}(\theta),
\end{gathered}
$$

where $\tau_{i j}^{-1}\left(\theta, \theta^{\prime}\right)$ and $\eta_{n, i \bar{j}}(\theta)$ depend on the type of impurities (intra- or inter-plane) -see 10], while $\eta_{n, i \bar{j}}(\theta)$ are solutions of a coupled set of integral equations (w.r.t. $\theta)$. In the presence of inter-plane impurities (the case $I)$, one has $\tau_{i j}^{-1}\left(\theta, \theta^{\prime}\right)=\overline{\delta_{i j} N_{i}(\theta)} U\left(\theta, \theta^{\prime}\right)$, where $N_{i}(\theta)$ are the fully symmetric angle dependent band $(i=1,2)$ density of states on the Fermi surface. Note, this kind of impurities does not affect isotropic $s$-wave pairing - see [10], but it does affect $d$-wave pairing [12]. In the case $I I$ of the intra-plane impurities one has $\tau_{i j}^{-1}\left(\theta, \theta^{\prime}\right)=U_{i}\left(\theta, \theta^{\prime}\right) N_{j} \overline{(\theta) \text { with } U_{1}}\left(\theta, \theta^{\prime}\right)=U_{2}\left(\theta, \theta^{\prime}\right)$. Note, the $s$-wave inter-layer pairing is strongly affected by these impurities, due to the breaking of the local reflection symmetry between layers $\mid 10$ - transitions between symmetric and antisymmetric band occur.

Let us analyze the case $I I$, which is frequently realized in the $\mathrm{YBa}_{2} \mathrm{Cu}_{3} \mathrm{O}_{7-x}$ family. A very important ingredient of our theory are strong correlations, for which it has been shown that in $\tau^{-1}\left(\theta, \theta^{\prime}\right)$ only $s$ - and $d$-channels dominate [1], [2], [3]. When generalized to the bi-layer model one has $\tau_{i j}^{-1}\left(\theta, \theta^{\prime}\right)=\Gamma_{s, i j}\left(\theta, \theta^{\prime}\right)+\Gamma_{d, i j} Y_{d}(\theta) Y_{d}\left(\theta^{\prime}\right)+\ldots$. Note, $\Gamma_{s, i j}\left(\theta, \theta^{\prime}\right)(>0)$ is fully symmetric and it holds $\Gamma_{s, i j}\left(\theta, \theta^{\prime}\right)>\Gamma_{d, i j} Y_{d}(\theta) Y_{d}\left(\theta^{\prime}\right)$, where $\Gamma_{d, i j}>0$. Their doping dependence is analyzed below. Let us assume that the fully symmetric scattering rates are weakly angle dependent, i.e. $\Gamma_{s, i j}\left(\theta, \theta^{\prime}\right) \simeq \Gamma_{s, i j}$. In this case eq.(3) is reduced to a set of algebraic equations for the critical temperature $T_{c}$

$$
\Delta_{i}=T_{c} \sum_{n} \lambda_{i \bar{j}} \frac{\eta_{n, \bar{j} \bar{k}} \Delta_{\bar{k}}}{\left|\omega_{n}\right| \eta_{n}}
$$

where $\eta_{n}=1+\left(\Gamma_{s, 11}+\Gamma_{s, 22}\right) /\left|\omega_{n}\right|, \eta_{n, i j}=\delta_{i j}+\Gamma_{d, j j} /(\mid$ $\left.\omega_{n} \mid+\Gamma_{s}-\Gamma_{d}\right), \Gamma_{s}=\Gamma_{s, 11}+\Gamma_{s, 22}$, and $\Gamma_{d}=\Gamma_{d, 11}+\Gamma_{d, 22}$. $E q .(7)$ contains reach physics which depends on the values and signs of the coupling constants $\lambda_{i j}$ see [12]. However, in order to illustrate the basic effect of strong correlations we simplify the problem by assuming that the inter-layer pairing is negligible, i.e. $\Delta_{\perp}(\vec{p}) \approx 0$ and $\Delta_{1} \approx \Delta_{2} \approx \Delta_{\|}$. Then eq.(7) is reduced to

$$
\ln \frac{T_{c}}{T_{c}^{0}}=\left[\psi\left(\frac{1}{2}\right)-\psi\left((1-\beta) x+\frac{1}{2}\right)\right],
$$

where $\beta=\left(\Gamma_{d} / \Gamma_{s}\right)<1, x=\Gamma_{s} / 4 \pi T_{c}$ and $T_{c}^{0}=$ $1.13 \omega_{c} \exp \{-1 / \lambda\}, \lambda=\lambda_{11}+\lambda_{12}$. Similar equation has been obtained also in 13. It tells us that the pair-breaking effect of nonmagnetic impurities is strongly reduced if the scattering potential contains a large anisotropic part which is compatible with the pairing amplitude. It follows from eq.(8) that in the presence of an anisotropic scattering $(\beta \neq 0) d$-wave pairing is more robust than in the case of the isotropic one $(\beta=0)$. This robustness is reflected in the decrease of the slope of $T_{c}(x)$ at $x=0$ by factor $(1-\beta)$. It should be stressed that the order parameter keeps its $d$-wave form, i.e. $\Delta(\theta) \approx \Delta_{d} Y_{d}(\theta)$ and $\langle\Delta(\theta)\rangle=0$.

The above simple analysis would not deserve to much attention if we would not be able to find the range of the parameter $\beta$, as well as the origin for its large value in $H T S$. Luckily, the doping dependence of $\beta(\delta)$ can be extracted from the theory of strong correlations 1], [2, [3], where for $\delta \leq 0.2$ the parameter $\beta$ becomes of the order of one(!), while at large doping $(1-\delta \rightarrow 0)$ one has $\beta \ll 1$-see Fig.1. The latter value leads to a rapid decrease of $T_{c}$ by increasing $\Gamma_{s}$. These results tell us that $d$-wave pairing in the underdoped and optimally doped $H T S$ materials is robust in the presence of impurities, while it is much less robust in the overdoped systems see Fig.1. 


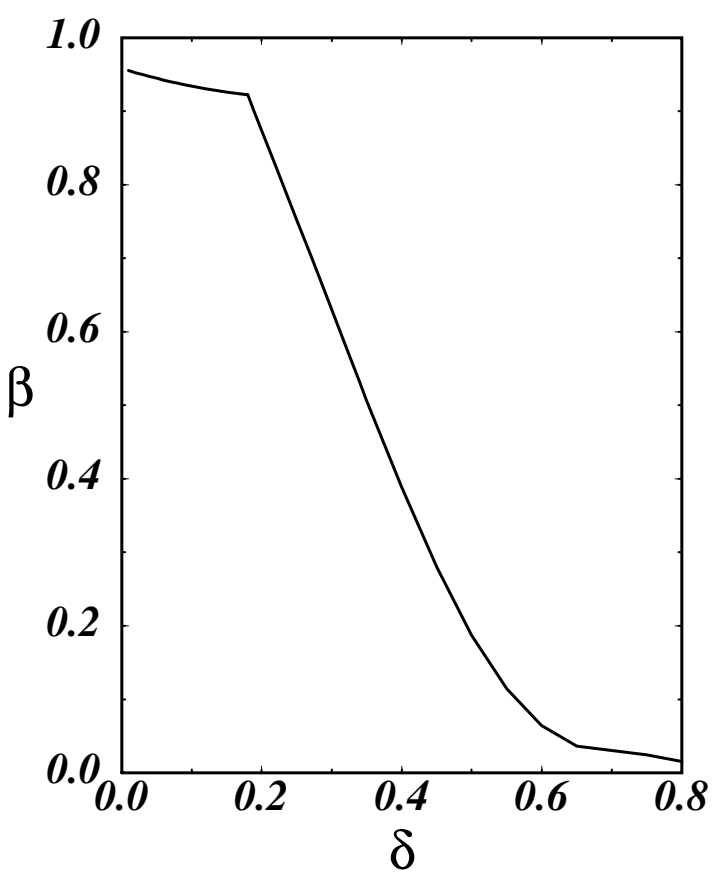

FIG. 1. The anisotropic scattering parameter $\beta$ as a function of the doping concentration $\delta$ based on the work [2].

The average pair-breaking parameter $\Gamma_{s}$ is extracted from the measurements of the residual resistivity $\rho_{i}=$ $4 \pi / \tau_{t r} \omega_{p l}^{2}$, which is due to the substitutional defects $(C u \rightarrow Z n, Y \rightarrow \operatorname{Pr})$ or due to the ion $\left(N e^{+}\right)$or electron damaging of HTS materials - see [6], [7]. The plasmon frequency $\omega_{p l}$ is extracted from the slope $\alpha$ in the linear resistivity $\rho(T)=\rho_{i}+\alpha T$, where $\left(4 \pi / \omega_{p l}^{2}\right)=\alpha / 2 \pi \lambda_{t r}$ and $\lambda_{t r}$ is the transport coupling constant. The realistic guess for $\lambda_{t r}$ is $\lambda_{t r} \approx 0.3$ [15]. However, it is not correct to replace $\Gamma_{s}$ by $1 / \tau_{t r}$ because for the latter backward processes dominate. It turns out that in the presence of strong correlations one has $\Gamma_{s}=p \cdot 1 / \tau_{t r}$, i.e. $\Gamma_{s}$ $=2 p \pi \lambda_{t r}\left(\rho_{i} / \alpha\right)$ and for/below optimal doping $\delta \leq 0.2$ it is obtained $p \approx 2$ and $\beta \geq 0.8$ [2], [3].

We discuss here three scenarios for the suppression of the measured slope $\left(d T_{c} / d x\right)_{x=0}\left(x=\rho_{i} / \alpha\right)$, which are compared to the prediction of the $A-G$ theory [9], [7] for $d$-wave pairing with isotropic impurity scattering.

1) $d$-wave pairing $\left(\langle\Delta(\theta)\rangle_{\theta}=0\right)$ and anisotropic scattering $(\beta \neq 0)$

Based on the above analysis and from eq.(8) one has

$$
\left(d T_{c} / d x\right)_{x=0}=-p(1-\beta) \lambda_{t r} \pi^{2} / 8,
$$

where $x=\rho_{i} / \alpha$. The experimental value is

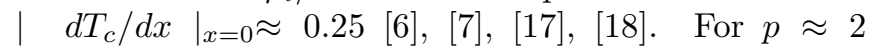
and $\lambda_{t r} \approx 0.3$ [15] one obtains $\beta^{\exp } \approx 0.85$. Such a large experimental value of $\beta$ is in accordance with the prediction of the theory of strongly correlated electrons [1], [2], [3]. This means that the present theory can consistently explain the experimental slope $\left|d T_{c} / d x\right|_{x=0}$ [6], [7] - see Fig.2.

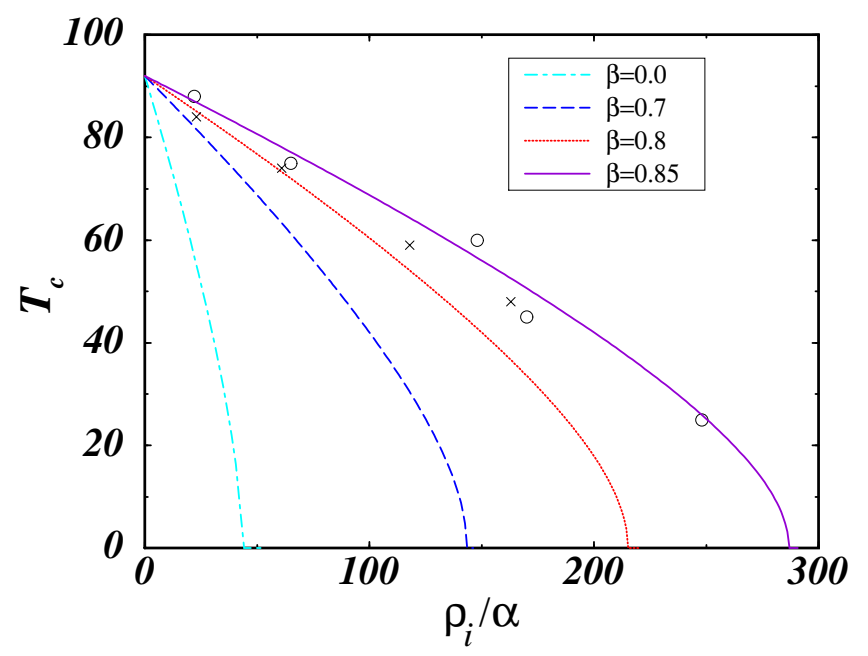

FIG. 2. The critical temperature $T_{c}[\mathrm{~K}]$ of $d-$ wave superconductor as a function of the experimental parameter $\rho_{i} / \alpha[\mathrm{K}]$. The case $\beta=0$ is the prediction of the standard $d-$ wave theory with isotropic scattering. The experimental data are given by crosses $-\mathrm{YBa}_{2} \mathrm{Cu}_{3-x} \mathrm{Zn}_{y} \mathrm{O}_{7-\delta}$ [17], and circles $-\mathrm{Y}_{1-y} \mathrm{Pr}_{y} \mathrm{Ba}_{2} \mathrm{Cu}_{3} \mathrm{O}_{7-\delta}$ [18].

2) Anisotropic pairing $\left(\langle\Delta(\theta)\rangle_{\theta} \neq 0\right)$ and isotropic scattering $(\beta=0)$

Based on eqs.(2)-(5) one obtains eq.(9), where $\beta \rightarrow$ $\beta_{\text {an }}=\langle\Delta(\theta)\rangle_{\theta}^{2} /\left\langle\Delta^{2}(\theta)\right\rangle-$ see $\left[14\right.$. For $\left|d T_{c} / d x\right|_{x=0} \approx$ 0.25 one has $\beta_{a n}^{e x} \approx 0.85$, i.e. $\langle\Delta(\theta)\rangle_{\theta} \approx 0.9 \sqrt{\left\langle\Delta^{2}(\theta)\right\rangle}$. This result means that a large $s-$ wave component appears in the order parameter, i.e. $\Delta(\theta)=\Delta_{s}+\Delta_{d} Y_{d}(\theta)$ and $\Delta_{d} \approx 0.5 \Delta_{s}$. This result is compatible with the experimental observation of the finite critical current in the $\mathrm{Pb}-Y \mathrm{BCO}$ Josephson contact along the $\mathrm{c}$ - axis [16], but it is incompatible with $d$-wave like pairing $\left(\left|\langle\Delta(\theta)\rangle_{\theta}\right| / \Delta_{\max } \ll 1\right)$ found in many experiments.

3) Anisotropic pairing $\left(\langle\Delta(\theta)\rangle_{\theta} \neq 0\right)$ and anisotropic scattering $(\beta \neq 0)$

In this case one should set $\beta \rightarrow \beta_{\text {eff }}$ in eq.(9), where $\beta_{\text {eff }}=\left(\beta \Delta_{d}^{2}+\Delta_{s}^{2}\right) /\left(\Delta_{d}^{2}+\Delta_{s}^{2}\right)$. Since $\beta_{\text {eff }}^{\exp } \approx 0.85$ one obtains $\Delta_{s}=2 \Delta_{d} \sqrt{1-\beta / 0.85}$. This means that finite $\beta$ suppresses the $s-$ wave component in $\Delta(\theta)$. In our opinion, the realistic situation for the optimal doping might be described by the case 1 ), or by 3 ) with $0.78<\beta<0.85$ and $0<\Delta_{s}<\Delta_{d} / 3$.

In conclusion, the effect of nonmagnetic impurities on $d$ - wave superconductivity in HTS materials is studied within the theory of strong correlations [1], 2], 3] which predicts: $(i)$ by lowering doping $\delta$ the forward scattering peak is developed in a nonmagnetic scattering where a large $d$-wave component is present in it; (ii) transport properties are renormalized in such a way that $1 / \tau_{t r}=\Gamma_{s} / p$, where $p \geq 2$ for $\delta<0.2$. Based on this theory, we have shown that the robustness of $T_{c}$ in $H T S$ systems in the presence of defects and impurities is due 
to: (a) an anisotropic scattering (due to strong correlations) and (b) the anisotropic pairing with $\langle\Delta(\theta)\rangle_{\theta}=0$ or $\left|\langle\Delta(\theta)\rangle_{\theta}\right| / \Delta_{\max } \ll 1$. The theory, which is generally formulated for the bi-layer model with intra- and inter-layer pairing and with intra- or inter-layer impurities, shows that the underdoped and optimally doped systems are robust in the presence of impurities, while the overdoped systems lose this robustness.

\section{Acknowledgments:}

One of us (M. L. K.) would like to thank Professors Michael Mehring, Ole K. Andersen and Lars Hedin for support. We thank Professors Konstantin Efetov and Vladimir Prigodin for discussions and important comments on the manuscript. Partial financial support by the Russian State Program "High-Temperature Superconductivity", Grant No 95065, and by the Russian Foundation for Fundamental Research, Grant No 96-0217527 , is acknowledged by one of us (V.O.)

[1] M. L. Kulić and R. Zeyher, Phys. Rev. B49, 4395 (1994)

[2] R. Zeyher and M. L. Kulić, Phys. Rev. B53, 2850 (1996)

[3] R. Zeyher and M. L. Kulić, Phys. Rev. B54, 8985 (1996)

[4] P. A. Lee and N. Nagaosa, Phys. Rev. B46, 5621 (1992)
[5] D. J. Scalapino, Phys. Rep. 250, 153 (1995)

[6] A. G. Sun, L. M. Paulius, D. A. Gajevski, M. B. Maple and R. C. Dynes, Phys. Rev. B50, 3266 (1994)

[7] S. K. Tolpygo, J.-Y. Lin, M. Gurvitch, S. Y. Hou, J. M. Philips, Phys. Rev. B53, 12454 (1996); ibid. 12462 (1996)

[8] An opposite effect of increased backward impurity scattering, due to the Kohn anomaly, on the $p$-wave pairing was studied in K. B. Efetov, Sov. Phys. JETP, 46, 1020 (1978)

[9] A. A. Abrikosov and L. P. Gorkov, Sov. Phys. JETP, 10, 593 (1961)

[10] U. Hofmann, J. Keller and M. L. Kulić, Z. Phys. B Condensed Matter $\underline{81}, 25$ (1990)

[11] R. Gajić, J. Keller and M. L. Kulić, Sol. State Comm. $\underline{76}, 717$ (1991)

[12] M. L. Kulić, R. Gajić and V. Oudovenko, in preparation

[13] G. Harán and A. D. S. Nagi, Phys. Rev. B54, 15463 (1996)

[14] P. C. Hohenberg, Sov. Phys. JETP, 18, 834 (1964); S. V. Pokrovsky and V. L. Pokrovsky, Phys. Rev. B54, 13275 (1996)

[15] M. Gurvitch and A. T. Fiory, Phys. Rev. Lett., 59, 1337 (1987)

[16] A. G. Sun, D. A. Gajevski, M. B. Maple and R. C. Dynes, Phys. Rev. Lett., 72, 2267 (1994)

[17] T.R. Chien, Z.Z. Wang and N.P. Ong, Phys. Rev. Lett., 67, 2088 (1991).

[18] J.J. Neumeier and M.B. Maple, Physica C191, 158 (1992). 